\title{
Don't fight the tape! Technical Analysis Momentum and Contrarian Signals as Common Cognitive Biases ${ }^{1}$
}

\author{
Piotr Zielonka², Wojciech Białaszek ${ }^{3}$, Paweł Biedrzycki ${ }^{4}$ Bartłomiej Dzik ${ }^{5}$
}

Submitted: 26.02.2020. Accepted: 21.04.2020

\section{Abstract}

Purpose: Stock market participants use technical analysis to seek trends in stock price charts despite its doubtful efficiency. We tested whether technical analysis signals represent typical and common cognitive biases associated with the continuation or reversal of the trend.

Methodology: We compared investors' opinions about the predictive power of technical analysis signals grouped into five conditions: real technical analysis signals associated with trend continuation (real momentum signals) or trend reversal (real contrarian signals), fake momentum or fake contrarian signals, and fluctuation signals.

Findings: Investors assigned larger predictive power to real and fake signals associated with trend continuation than to signals associated with trend reversal. Fake signals, which represented cognitive biases, elicited similar predictions about trend continuation or reversal to real technical analysis signals.

Originality: Market players assess momentum signals to have greater predictive power than contrarian signals and neutral signals to have the least predictive power. These results are independent of whether technical analysis signals were well-known to investors or made up by experimenters. The hardwired propensity of our brains to detect patterns combined with the non-natural environment of the stock market creates the illusion of expertise that is not easy to dispel.

Keywords: technical analysis, pattern recognition, cognitive biases, momentum, contrarian

JEL: D91, G41

\footnotetext{
1 Preparation of this manuscript was supported by the National Science Centre Poland under Grant 2015/19/D/HS6/00770 and by subsidies from MNiSW, decision number WP/2018/A/05 (Faculty of Psychology, SWPS University of Social Sciences and Humanities).

2 Corresponding author, Warsaw University of Life Sciences, 166 Nowoursynowska St., 02-787 Warsaw, Poland; e-mail: piotr _zielonka@ sggw.edu.pl.

3 SWPS University of Social Sciences and Humanities, Institute of Psychology, DecisionLab, 19/31 Chodakowska St., 03-815 Warsaw, Poland; e-mail: wbialaszek@swps.edu.pl; https://orcid.org/0000-0002-4672-4376.

4 Kozminski University, 59 Jagiellońska St., 03-301 Warsaw, Poland; e-mail: P.Biedrzycki@strefainwestorow.pl.

5 Kozminski University, 59 Jagiellońska St., 03-301 Warsaw, Poland; e-mail: bdzik@wp.pl.
} 


\section{Introduction}

The human brain has evolved in order to recognize a variety of patterns, both spatial and temporal. Pattern recognition was essential for survival, especially in terms of food gathering, hunting, and avoiding predators. It could be demonstrated that a hostile environment, in which our ancestors struggled to survive, punished type II pattern recognition errors (false negatives) much more severely than type I errors (false positives). While a single false negative could easily cost one's life, a false positive result just ended up wasting time and inducing fatigue. This strong asymmetry encouraged the 'over-calibration' of pattern recognition sensitivity to the false positive side of the spectrum. A good real-world example of this over-calibration is people's propensity to see human faces in oddly-shaped objects like stains or tree rings, while it is extremely unlikely to mistake a human face for any other object.

Pattern recognition sensitivity is a part of a wider human propensity for causal reasoning instead of accepting the random character of the environment. People tend to view human-like agency - like deities or supernatural beings in some cultures - as responsible for many environmental phenomena; a phenomenon labeled hyperactive agent-detection device (Barret, 2000), which has been used to explain the origin of early religious beliefs.

Recognizing temporal patterns is as important as recognizing spatial patterns. In both natural and social life, humans encounter cyclicality and repetitiveness. As long as the environment remains stable, one may assume that natural phenomena, instinct-driven animals, and rational social actors today behave more or less in the same manner as they behaved yesterday and the day before yesterday. The ability to recognize temporal patterns has become more important along with advances in human civilization, meaning the transition from hunter-gatherer to agricultural societies. The temporal pattern observed in nature and society overwhelmingly features positive autocorrelations (trend continuation, momentum); that is, subsequent changes have the same sign.

For example, the length of daytime alternates its pace only twice a year, and for the remaining 363 days features a stable increasing or decreasing trend. Many human characteristics are supposed to change steadily with only one point of alternation during the lifetime, such as physical strength or mental ability; excluding short-term distortion caused by e.g. illness. It is notable that the phenomena less compatible with this general pattern due to a relatively quick alternation cycle, such as moon phases, were commonly seen as having something to do with the supernatural (Lo and Hasanhodzic, 2010). 
Thus, our brains have been evolutionarily conditioned (one could even say 'hardwired') to assume - prior to assessing any evidence - that a temporal pattern features positive autocorrelations (Wilke and Clark Barrett, 2009). Negative autocorrelations (trend reversal) are almost nonexistent in nature and social life. Even if they do occur, they usually amount to some compound phenomena such as regression to the mean. Yet this seems a highly counterintuitive phenomenon; understanding it requires some deliberation.

Instead of temporal patterns with negative autocorrelation in nature, we face temporal patterns without any autocorrelation, that is, a series of random independent events. Randomness and statistical independence are abundant in nature - for instance, the Brownian motion of particles - but they also occasionally find their way into the macro scale. In fact, from the very beginning, humans were exposed to the most basic example of a random process with IID (independent and identically distributed random variables), very much resembling a series of fair coin flips: the male-female birth order. Of course, for a given individual, the sample size comprised of one's children was very small by the standards of statistical inference (yet two-digit numbers were not unusual), but one could see over the course of a lifetime that the sample of offspring - for both human and livestock - in the entire clan/village/town might have been reasonably large. Nevertheless, despite this common exposure and the high relevance of procreation process in daily life, the randomness associated with birth orders was fairly universally rejected. Instead, ancient cultures developed dozens of different methods to predict the gender of an unborn child, all sharing two common characteristics: (1) being totally ineffective, and yet (2) prevailing for generations, even centuries. The reason why people failed to learn about the nature of randomness and statistical independence from birth order could be twofold. First, concentration on mastering the recognition of autocorrelated events simply 'overruled' any alternative hypotheses or suppressed the perception of counterevidence. Second, for the majority of human evolutionary history, it was the number of children that counted, the birth order and male-to-female ratio being of secondary importance. Family planning based on the ovulation cycle, while not as reliable as modern contraception, definitely operates above the level of pure randomness, as it was based on an actual cyclical phenomenon. On the other hand, folk methods of predicting (or sometimes influencing) the sex of the offspring could not beat a pure chance guessing game, yet could persist unquestioned for centuries, such as the Chinese Gender Chart from the Qing Dynasty (Your Chinese Astrology, 2019). Thus, human fertility, involving both purely random elements (the sex of the offspring) and some cyclicality (ovulation cycle), gives rise to something that could be described as the great-grandparent of technical analysis. Technical analysis is a discipline investors use to identify trading opportunities by analyzing trends in price and volume movements. 
The invention of gambling gave rise to another milestone in human exposure to pure randomness. While in the case of birth order, humans were simply lacking the conceptual model and thus resorted to superstition, gambling by its very definition was 'a game of chance.' But then again, the urge to find patterns overruled more nuanced considerations, and gamblers - to their despair - have tried to find patterns in series of dice rolls or spins of a roulette wheel. Studies on the perception of randomness (such as streaks of fair coin tosses) reveal that people 'expect' random processes to feature many alternations, such that typical longer streaks of heads/tails in fair coin tosses are deemed 'suspicious' (Bar-Hillel and Wagenaar, 1991). The desire for self-correction in a random process may additionally stem from cognitive bias known as "belief in the law of small numbers" (Tversky and Kahneman, 1971). The limited capacity of shortterm memory may also play a role (Hahn and Warren, 2009).

The same over-adjustment probably applies to the expectation about a random walk. It could be easily demonstrated that the simplest random walk (either +1 or -1 with equal probabilities) is frequently located either totally above or totally below the X-axis. After the first step, the value is either +1 or -1 , and the expected value of any subsequent future steps is always zero (for technical discussion, see Epstein, 1977, ch. 4). However, common imagination hints at a different pattern: the one that crosses the $\mathrm{X}$-axis several times, resembling a sinusoid. The implications for technical analysis become obvious: a 'typical' random walk will, in fact, be assessed as a process featuring 'trends,' 'supports,' and 'resistances;' all of these contributing to a 'lesser than expected' number of alternations. Research by Roney and Trick (2009) also suggests that the propensity to detect "streaks" is reinforced when perception is focused on a human involved in generating a random process (e.g. someone flipping the coin) rather than the process itself. This means that any activity involving human agency - such as stock trading - will likely trigger the default mode of detecting positive autocorrelations.

Human misunderstanding of randomness not only demonstrates itself in biased perceptions but also affects strategic decisions: in simple experimental games, in which optimal strategy involves randomization, human subjects are outperformed by chimpanzees (Martin et al., 2014). However, we should note that some ancient cultural practices, like certain divinations, may actually evolve as a countermeasure to human biases in pattern recognition (for discussion, see Henrich, 2015, ch. 7). Moreover, while biases in the perception of randomness are abundant, researches should also double-check whether a given naturalistic setting actually involves independent random variables. For many years, the belief in 'hot hand' in basketball (Gilovich, Vallone, and Tversky, 1985) was considered a cognitive illusion, yet recent fine-tuned statistical analyses eventually proved that the phenomenon is real (Miller and Sajurjo, 2019). 
Stock prices are really hard to predict. From the normative perspective, it is impossible to systematically predict the future changes in stock prices by analyzing time series of past prices (Malkiel and Fama, 1970; Fama, 1991). Nevertheless, the strong propensity to detect patterns in time series of stock prices makes technical analysis a very tempting alternative to the passive acceptance of the unpredictable.

While both the propensity to look for patterns with positive autocorrelation and biased perception of randomness may explain the popularity of technical analysis, we should note that investors do not follow signals unconditionally. Such a 'rigid' approach would result in an extreme case of herding behavior on the stock market and subsequently create disastrous bubbles. Technical analyses produce hints rather than infallible guidance, and they leave some room for ambiguity. Depending on the assumed perspective and the level of finesse in interpretation, one could derive several different - and possibly contradictory - signals from a sufficiently complex pattern (De Bondt, 1993). This gives the investor merely the necessary level of discretion over their choices and allows for the sporadic utilization of technical analysis as an ex-post rationalization of the buying/selling decision (Shafir, Simonson, and Tversky, 1993). The strongest version of this process may in fact involve reverse causation, i.e. the unconscious belief that the individual's decision to buy a particular stock is a diagnostic of the upcoming increase in its value (Quattrone and Tversky, 1995). On the other hand, the correlations between the propensity to rely on technical analysis and different measures of overconfidence are moderate at best (Kubińska et al., 2018), which suggests that some basic cognitive factors are at play rather than individual motivational processes. We should mention that some professional investors use technical analysis even though they know it is not as effective as they would like. Facing high costs of acquiring fundamental information, investors treat technical analysis as an inexpensive form of obtaining information (Menkhoff, 2010), even if this information is not fundamental at all.

To sum up, technical analysis appears to be a very attractive framework for stock market investors: it fits very well with our hardwired propensity to detect patterns (especially a continuation of trends) and seems helpful in the ex-post rationalization of investment decisions. However, it only provides a rationale for perpetuating biases in the perception of randomness.

\section{Hypotheses}

Predictions involving the continuation of trends are most common in natural and social life and possibly hardwired in our brains (for an overview from an evolutionary 
perspective, see Scheibehenne, Wilke, and Todd, 2011). Laboratory experiments on the perception of randomness point to a belief in short-term momentum (assuming positive autocorrelation) as a dominant cognitive strategy (Tyszka et al., 2008). Predicting the continuation of trends engages minimal cognitive resources, compared to attempts to detect trend reversal (Tyszka et al., 2017).

Thus, we expected that the technical analysis signals predicting the continuation of the trend in market prices (the 'momentum' signals) would receive the strongest judged predictive power. On the other hand, the movement of prices resembling the common idea of random noise (like a zigzag-style pattern) should be assessed by the investors as the least predictive ('fluctuation' signals). Finally, we expected that the technical analysis signals predicting the reversal of the trend ('contrarian' signals) will fall somewhere in between: they will still be acknowledged because of a general propensity to recognize patterns, but they will receive less predictive power than momentum signals as they are atypical in nature. Our deliberations could be operationalized into two main hypotheses:

H1: Technical analysis real momentum signals will be assigned stronger predictive power than technical analysis of real contrarian signals.

H2: Investors will assign stronger predictive power to both technical analysis momentum signals and technical analysis contrarian signals than to 'fluctuation' signals, i.e. to patterns not associated with cognitive biases.

Furthermore, because real investment behavior frequently embeds post-hoc rationalizations of buying/selling decisions and the set of technical analysis signals is large and fluctuating, we expect that the assessment of the strength of technical analysis signals mostly depends on its resemblance of the general 'momentum'/'contrarian' pattern like 'the fall is expected after the rise and plateau.' In other words, investors by no means rely on a closed set of 'officially established' signals. Therefore, one should be able to "make up" brand new technical analysis signals that will still be considered meaningful for the investors (think of 'the dome and turrets' instead of 'head and shoulders'). We call such made-up signals 'fake momentum' and 'fake contrarian,' and we formulate the following auxiliary hypothesis:

H3: There will be no difference in judged predictive power assigned to 'fake' technical analysis signals and their respective 'real' counterparts. 


\section{Method}

\section{Participants}

Participants were stock market investors, clients of a large investment Internet portal, who had knowledge of and used technical analysis. The link from email was followed by 339 users ( 30 females, 309 males; mean age 44.8 years, SD = 12.7). We included only full responses in the analyses, i.e. participants who answered all questions. The final sample consisted of 214 respondents.

\section{Materials and Procedure}

Participants first acknowledged the informed consent form and, after agreeing to participate in the research, answered questions about gender and age. Prior to being presented with the questions in Table 1, participants received the following written instructions (presented in Polish):

Below, you will find a list of technical analysis signals of future price movements. Please rank each signal on the scale $-3,-2,-1,0,+1,+2,+3$, depending on your expectations regarding the behavior of the WIG Index in the next few weeks after the signal is detected. The higher the rank, the greater the expected increase. Thus, the rank of -3 means that you expect a significant decline and, alternatively, the rank of +3 foretells a substantial increase. The rank of " 0 " means that you consider the signal to be irrelevant for the prediction of WIG Index behavior. You may also choose the option "I don't know."

The order of questions (Table 1) was randomized once before the experiment, and then kept constant so that each participant answered questions in the same order, but the experimenters did not deliberately set the order. Each question was assigned to one of the five independent categories: (1) fluctuation signal's condition, (2) real contrarian and (3) fake contrarian signals, and (4) real and (5) fake momentum signals. The average scores within each category formed their respective indices, representing the strength of belief in a given type of signal (e.g. 'the strength of belief in fake contrarian signals'). These statistics are later referred to as 'fluctuation score,' 'fake contrarian score,' and so on.

Items $1,4,9,10$, and 18 were assigned to negative price movements (price going down): for items 1, 4, and 10 directly on the basis of technical analysis, for items 9 and 18 on the basis of our assumptions about the similarity to established signals. Therefore, to assure comparability, the answers to these questions were coded with reversed signals. 
The answer "I don't know" was implemented in the questionnaire to ensure the quality of the data, because there were indeed correct and incorrect answers.

Table 1. Items used to measure the strength of belief in: fluctuation signals (FS), real contrarian signals (RCS), fake contrarian signals (FCS), real momentum signals (RMS), and fake momentum signals (FMS)

\begin{tabular}{|c|c|c|}
\hline Item \# & Item content & Type of sign \\
\hline 1 & $\begin{array}{l}\text { A Head and Shoulders pattern formed by the WIG Index after a major } \\
\text { upward trend. }\end{array}$ & RCS \\
\hline 2 & A series of alternative low and high turnovers in consecutive sessions. & FS \\
\hline 3 & $\begin{array}{l}\text { All stock market indices increase three days in a row after the resolution } \\
\text { of government crisis. }\end{array}$ & FMS \\
\hline 4 & Another index rebounds at the resistance level. & RCS \\
\hline 5 & Decreasing price volatility within a horizontal trend. & FS \\
\hline 6 & The WIG Index rising $10 \%$ within a week along with increasing turnover. & FMS \\
\hline 7 & The WIG Index forming longer and longer plateaus. & FS \\
\hline 8 & Increasing WIG Index breaks out through consecutive resistance levels. & RMS \\
\hline 9 & $\begin{array}{l}\text { The WIG Index fails the second time in a row to test four-fifth of the latest } \\
\text { maximum. }\end{array}$ & FCS \\
\hline 10 & Falling WIG Index breaks out through consecutive support levels. & RMS \\
\hline 11 & $\begin{array}{l}\text { The typical sinusoid pattern of the WIG Index initiated within a horizontal } \\
\text { trend. }\end{array}$ & FS \\
\hline 12 & $\begin{array}{l}\text { After a series of WIG Index falls, favorable news about the industrial output } \\
\text { is disclosed. }\end{array}$ & FCS \\
\hline 13 & $\begin{array}{l}\text { The WIG Index is noticeably breaking through strong psychological anchors, } \\
\text { such as } 30 \mathrm{~K} \text { pts or } 40 \mathrm{~K} \text { pts. }\end{array}$ & RMS \\
\hline 14 & The increasing volatility of the WIG Index within a horizontal trend. & FS \\
\hline 15 & $\begin{array}{l}\text { After several month of WIG Index downtrend, numerous "buy" } \\
\text { recommendations are issued by brokerage houses. }\end{array}$ & FCS \\
\hline 16 & Alternative up and down breakouts from the horizontal trend. & FS \\
\hline 17 & After the WIG Index falls, there appears very large excessive demand. & RCS \\
\hline 18 & The WIG Index falls in parallel to the governmental crisis. & FMS \\
\hline
\end{tabular}

Source: own elaboration. 


\section{Results}

We analyzed the data using the repeated measures ANOVA (multivariate approach, reported index: Pillai's trace). There were significant differences between fluctuation and trend-based (fake/real $\times$ contrarian/momentum) experimental conditions $\mathrm{F}(4 ; 207)$ $\left.=73,202 ; p<.001 ; \eta^{2}=0.586\right)$. The mean scores with 95\% confidence intervals for all conditions are presented in Figure 1.

\section{Figure 1}

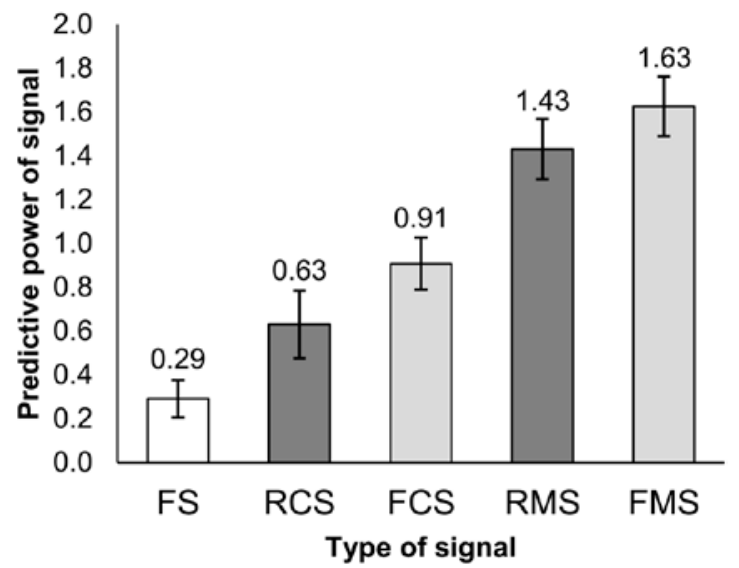

Average scores and $95 \%$ confidence intervals for all conditions in the study. All differences between mean scores are statistically significant, except for the difference between real momentum and fake momentum conditions (see text for reference). Abbreviations: FS - fluctuation signals, RCS - real contrarian signals, FCS - fake contrarian signals, RMS - real momentum signals, and FMS - fake momentum signals. The predictive power of signal ranges from -3 to +3 , although the values on the vertical axis were adjusted to $0-2$ range to make the differences more visible.

Source: own elaboration.

We conducted post-hoc comparisons with the Šidák correction for multiple comparisons. The only statistically insignificant difference was the one between the two highest mean scores: for real momentum signals and for fake momentum signals $(\mathrm{p}=0.066)$. The difference between real momentum and real contrarian mean scores was highly significant ( $\mathrm{p}<0.001$ ), thus supporting hypothesis H1. Hypothesis $\mathrm{H} 2$ also received strong support: all differences between fluctuation and other conditions were statistically significant, with significant difference between fluctuation and real contrarian mean scores at the level of $p=0.026$, while the other three trend conditions presented difference as highly significant $(\mathrm{p}<0.01)$.

The evidence collected also supports hypothesis H3: the differences between real and fake dimensions were very small. The strong version of the hypothesis has been con- 
firmed for momentum conditions; as mentioned above, the differences between real momentum scores and fake momentum scores were statistically insignificant. Taken together, the available evidence for $\mathrm{H} 3$ and $\mathrm{H} 2$ could be interpreted as a very general propensity of the investors to detect trends while ignoring information unrelated to trend continuation or reversal.

The analysis of responses also revealed modest yet significant positive correlations for almost all pairs of trend conditions; the only exception being that fake momentum was insignificantly correlated with real contrarian. The correlations are listed in Table 2. The strongest correlations observed were between real and fake momentum conditions, which could be expected on the basis of hypotheses H1 and H3. The scores for the fluctuation condition were not significantly correlated with scores for any trend condition, giving some further support for hypothesis $\mathrm{H} 2$. The observed correlations support the idea of investors (or humans in general) as persistent 'pattern seekers.'

Table 2. Pearson's correlation among scores for the strength of belief in terms of five types of signals

\begin{tabular}{|l|c|c|c|c|}
\hline & $\begin{array}{c}\text { fake } \\
\text { contrarian }\end{array}$ & $\begin{array}{c}\text { real } \\
\text { contrarian }\end{array}$ & $\begin{array}{c}\text { fluctuation } \\
\text { signals }\end{array}$ & $\begin{array}{c}\text { fake } \\
\text { momentum }\end{array}$ \\
\hline fake contrarian & 1 & & & \\
\hline real contrarian & $0.155^{\star}$ & 1 & 1 & \\
\hline fluctuation signals & -0.065 & -0.064 & -0.015 & 1 \\
\hline fake momentum & $0.290 * \star \star$ & 0.095 & -0.063 & $0.465^{\star * *}$ \\
\hline real momentum & $0.152^{\star}$ & $0.200 * \star$ & & \\
\hline
\end{tabular}

$* \mathrm{p}<.05, * * \mathrm{p}<.01, * * * \mathrm{p}<.001$

Source: own elaboration.

\section{Discussion}

The human propensity to detect patterns is essential to our survival and success as a species. Especially the propensity to detect streaks (a series of events with positive autocorrelation) seems evolutionarily hardwired, because false positives generated during such a process are less costly than potential false negatives (Barret, 2000, p. 31).

As a result, the propensity to assume trend continuation is highly resistant to contrary evidence (Scheibehenne et al., 2011) and requires minimal cognitive resources to operate 
(Tyszka et al., 2017). Nevertheless, such a propensity may backfire in an artificial environment, such as the stock exchange. Technical analysis resembles strategies (momentum and contrarian) used by regular casino gamblers to predict events that are, in fact, essentially unpredictable (Zielonka, 2004).

We speculated that technical analysis signals that predict the continuation of a trend (momentum) will be assessed by market players as having larger predictive power than contrarian signals. The hypothesis was confirmed. The contrarian signals that predict the reversal of trends elicited weaker responses than momentum ones, because trend reversals are uncommon in nature and human brains are not so well-equipped to detect them. Nevertheless, contrarian signals still elicited a stronger response than fluctuation ones (line sinuous pattern), thus confirming that some deliberative process is involved in the interpretation of price patterns.

While the results obtained regarding momentum and contrarian and fluctuation signals could be viewed as highly predictable or even trivial, the second dimension of our experiment - which involved comparing fake and real technical analysis signals - yielded nontrivial results. One may argue that comparing the real and fake signals involves conflicting motives: the appeal to the authority of established signals versus the attractiveness of the novelty of made-up signals. The made-up signals may thus resemble insider knowledge to some degree, and by following them, one could hope to 'beat the market.'

Our results suggest that these conflicting motives mostly cancel each other out, with 'novelty' having a small edge over 'authority.' An alternative explanation may suggest that investors treat technical analysis as a kind of vague art, and simply do not care about differentiating (or even cannot differentiate) between fake and established signals. In our opinion, this aspect of technical analysis is definitely worth further investigation.

Another issue that deserves further inquiry is whether our results would be replicated for professional traders. Professional investors not only differ from amateurs in experience but also in the access to information (the ability to use fundamental analysis) and agency (investing one's own vs. other people's money can make a difference). Experienced traders exhibit less cognitive biases than inexperienced ones (List, 2003), although these results were obtained in the commodities market (memorabilia, sportscards) and thus may not translate to stock market trading.

This also implies that it is easy to sow confusion among investors by inventing a technical analysis signal on the fly, as long as they resemble established signals. Moreover, 
technical analysis signals resemble a set of typical cognitive inclinations presented in the style typical for financial investors.

In conclusion, we found that market players assess momentum signals to have greater predictive power than contrarian signals, and fluctuation signals to have the least predictive power. These results are independent of whether technical analysis signals were well-known to investors or made up by experimenters. The hardwired propensity of our brains to detect patterns combined with the non-natural environment of the stock market creates the illusion of expertise that is not easy to dispel. Even though technical analysis is inefficient as a method to beat the market, we should also keep in mind that it is harmless. Studying the assessment of stock market players enriches our knowledge about the persistence of cognitive biases, but whether we should strive to debunk such biases in the general population of market players remains a separate research objective.

\section{Conflict of Interest}

The authors declare that the research was conducted in the absence of any commercial or financial relationships that could be construed as a potential conflict of interest.

\section{Data availability statement}

Datasets generated and analyzed during the current study are available from the corresponding authors on reasonable request.

\section{References}

Bar-Hillel, M. and Wagenaar, W.A. (1991). The Perception of Randomness. Advances in Applied Mathematics, 12(4), 428-454. https://doi.org/10.1016/0196-8858(91)90029-I.

Barret, J.L. (2000). Exploring the natural foundations of religion. Trends in Cognitive Sciences, 4(1), 29-34. https://doi.org/10.1016/S1364-6613(99)01419-9.

De Bondt, W.F.M. (1993). Betting on trends: Intuitive forecasts of financial risk and return. International Journal of Forecasting, 9, 355-371.

Epstein, R.A. (1977). The Theory of Gambling and Statistical Logic. London, UK: Academic Press.

Fama, E.F. (1991). Efficient Capital Markets: II. The Journal of Finance, 46(5), 1575-1617. https://doi.org/10.1111/j.1540-6261.1991.tb04636.x.

Gilovich, T., Vallone, R., and Tversky, A. (1985). The hot hand in basketball: On the misperception of random sequences. Cognitive Psychology, 17(3), 295-314. https://doi.org/10.1016/0010-0285(85)90010-6.

Hahn, U. and Warren, P.A. (2009) Perceptions of Randomness: Why Three Heads Are Better Than Four. Psychological Review, 116(2), 454-461. https://doi.org/10.1037/a0015241.

Henrich, J. (2015). The Secret of Our Success How Culture Is Driving Human Evolution, Domesticating Our Species, and Making Us Smarter. Princeton, Princeton University Press.

Kubińska, E., Czupryna, M., Markiewicz, Ł., and Czekaj, J. (2018). Technical analysis gives you courage, but not money - on the relationship between technical analysis usage, overconfidence 
and investment performance. Argumenta Oeconomica, 40(1), 317-344. https://doi.org/10.15611/aoe.2018.1.14.

List, J.A. (2003). Neoclassical Theory Versus Prospect Theory: Evidence from the Marketplace. Econometrica, 72(2), 615-625. https://doi.org/10.3386/w9736.

Lo, A.W. and Hasanhodzic, J. (2010). The Evolution of Technical Analysis: Financial Prediction from Babylonian Tablets to Bloomberg Terminals. Hoboken, NJ: Wiley.

Martin, C.F., Bhui, R., Bossaerts, P., Matsuzawa, T., and Camerer, C. (2014). Chimpanzee choice rates in competitive games match equilibrium game theory predictions. Scientific Reports, 4(5182), 1-6. https://doi.org/10.1038/srep05182.

Malkiel, B.G. and Fama, E.F. (1970). Efficient Capital Markets: A Review of Theory and Empirical Work. The Journal of Finance, 25(2), 383-417. https://doi.org/10.1111/j.1540-6261.1970.tb00518.x .

Menkhoff, L. (2010). The Use of Technical Analysis by Fund Managers: International Evidence. Journal of Banking \& Finance, 34(11), 2573-2586. https://doi.org/10.1016/j.jbankfin.2010.04.014.

Miller, J.B. and Sanjurjo, A. (2019). Is It a Fallacy to Believe in the Hot Hand in the NBA Three-Point Contest? IGIER Working Paper No. 548. https://doi.org/10.2139/ssrn.2611987.

Quattrone, G.A. and Tversky, A. (1995). Self-deception and the voter's illusion. In: J. Elster (ed.), The multiple self, 35-58. New York, NY: Cambridge University Press.

Roney, C.J.R. and Trick, L.M. (2009). Sympathetic magic and perceptions of randomness: The hot hand versus the gambler's fallacy. Thinking $\&$ Reasoning, 15(2), 197-210. https://doi.org/10.1080/13546780902847137.

Scheibehenne, B., Wilke, A., and Todd, P.M. (2011). Expectations of clumpy resources influence predictions of sequential events. Evolution and Human Behavior, 32(5), 326-333. https://doi.org/10.1016/j.evolhumbehav.2010.11.003.

Shafir, E., Simonson, I., and Tversky, A. (1993). Reason-based choice. Cognition, 49(1-2), 11-36. https://doi.org/10.1016/0010-0277(93)90034-S.

Tversky, A. and Kahneman, D. (1971) Belief in the law of small numbers. Psychological Bulletin, 76(2), 105-110. https://doi.org/10.1037/h0031322.

Tyszka, T., Markiewicz, Ł., Kubińska, E., Gawryluk, K., and Zielonka, P. (2017). A belief in trend reversal requires access to cognitive resources. Journal of Cognitive Psychology, 29(2), 1-15. https://10.1080/20445911.2016.1245195.

Tyszka, T., Zielonka, P., Dacey, R., and Sawicki, P. (2008). Perception of randomness and predicting uncertain events. Thinking $\&$ Reasoning, 14(1), 83-110. https://doi.org/10.1080/13546780701677669 .

Wilke, A. and Clark Barrett, H. (2009). The hot hand phenomenon as a cognitive adaptation to clumped resources. Evolution and Human Behavior, 30(3), 161-169, https://doi.org/10.1016/j.evolhumbehav.2008.11.004.

Your Chinese Astrology (2019). 2019 \& 2020 Chinese Baby Gender Prediction Chart. https://www.yourchineseastrology.com/calendar/baby-gender-predictor.htm (11.11.2019).

Zielonka, P. (2004). Technical analysis as the representation of typical cognitive biases. International Review of Financial Analysis, 13, 217-225. https://doi.org/10.1016/j.irfa.2004.02.007. 